\title{
A SURVEY ON STABLY DISSIPATIVE LOTKA-VOLTERRA SYSTEMS WITH AN APPLICATION TO INFINITE DIMENSIONAL VOLTERRA EQUATIONS
}

\author{
WALDYR M. OLIVA \\ The present paper is dedicated to Jaume Llibre, Luis Magalhães and \\ Carlos Rocha, on the occasion of their 60th birthdays
}

\begin{abstract}
For stably dissipative Lotka-Volterra equations the dynamics on the attractor are Hamiltonian and we argue that complex dynamics can occur. We also present examples and properties of some infinite dimensional Volterra systems with applications related with stably dissipative Lotka-Volterra equations. We finish by mentioning recent contributions on the subject.
\end{abstract}

2010 Mathematics Subject Classification: 34C25, 34K15.

Key words: Lotka-Volterra, stably dissipative systems, conservative systems, Poisson manifolds, reducible systems.

\section{Introduction}

Lotka-Volterra systems were introduced in the 1920s by A. J. Lotka [12] and V. Volterra [27] independently of one another in areas of chemistry and interaction of populations, respectively.

Volterra's researches in this field was strongly stimulated by conversations with the biologist Umberto D'Ancona from Siena. He investigated in considerably detail the association of two species, one of which (the predator) feeds on the other (the prey) and for this case we have the nowadays called Lotka-Volterra equations (see [29, p. 14, eq(4)]). Volterra's concern, however, was with associations of $n \geq 2$ species, and hence with the same number $n$ of differential equations. So, Volterra introduced the following system as a model for the competition of $n$ biological species

$$
\dot{x}_{j}=\varepsilon_{j} x_{j}+\sum_{k=1}^{n} a_{j k} x_{j} x_{k}, \quad j=1, \ldots, n .
$$


In this model, $x_{j}$ represents the number of individuals of species $j$ (so one assumes $x_{j}>0$ ), the $a_{j k}$ 's are the interaction coefficients, and the $\varepsilon_{j}$ 's are parameters that depend on the environment. For example, $\varepsilon_{j}>0$ means that species $j$ is able to increase with food from the environment, while $\varepsilon_{j}<0$ means that it cannot survive when left alone in the environment. One can also have $\varepsilon_{j}=0$ which means that the population stays constant if the species does not interact. This system (1) of differential equations is also called a Lotka-Volterra system.

In [28] Volterra considered a more general type of equation where some memory functions play the role of hereditary phenomena; hereditary here means, for instance, time of incubation or time of gestation of the female predator. When two individuals meet and one eats the other, the population of preys decreases immediately; on the other hand the population of predators takes a while to increase. This delay is interpreted as a constant number $r>0$ that appears in the system of equations (see (2)) and it is well known that we are dealing with retarded functional differential equations (RFDEs). In [5] we see the foundations and main results of the RFDEs. In particular, they define a flow in an infinite dimensional phase space. These more general equations introduced by Volterra are the following:

$$
\dot{x}_{j}(t)=x_{j}(t)\left[\varepsilon_{j}+\sum_{k=1}^{m} a_{j k} x_{k}(t)+\sum_{k=1}^{m} \int_{-r}^{0} x_{k}(t+\theta) F_{j k}(\theta) d \theta\right],
$$

where $\varepsilon_{j}, a_{j k}$ are constants and $x_{j}=x_{j}(t)>0$, for $j, k=1, \ldots, m$, and $F_{j k}(\theta)$ are the memory functions; the constant $r, 0<r \leq \infty$, is called the lag or delay of the equation. See [15] and [14] for cases where $0<r<\infty$ and $r=\infty$, respectively.

The dynamics of systems of type (1) are far from understood, although special classes of these Lotka-Volterra systems have been studied. We distinguish the following classes of systems of this type:

Definition 1.1. A Lotka-Volterra system with interaction matrix $A=$ $\left(a_{i j}\right)$ is called

(i) Cooperative (resp. Competitive) if $a_{j k} \geq 0$ (resp. $\left.a_{j k} \leq 0\right)$ for all $j \neq k$;

(ii) CONSERVATIVE if there exists a diagonal matrix $D>0$ such that $A D$ is skew-symmetric;

(iii) Dissipative if there exists a diagonal matrix $D>0$ such that $A D \leq 0$. 
Competitive systems and dissipative systems are mutually exclusive classes, except for the trivial case where $a_{j k}=0$. General results concerning competitive or cooperative systems were obtained by Smale [26] and Hirsch [7, 8] (for recent results see [30] and references therein). These systems typically have a global attractor consisting of equilibria and connections between them (see e. g. [7, Theorem 1.7]).

Dissipative systems have been less studied than competitive systems, although this class of systems goes back to the pioneer work of Volterra, who introduced them as a natural generalization of predator-prey systems (see [29, Chapter III]). For systems where predators and preys coexist there is empirical and numerical evidence that periodic oscillations occur. In fact, as it is well known, for any two dimensional predatorprey system, the orbits are periodic. But for higher dimensional systems the topology of orbits in phase space is much more complex, and understanding this topology is a challenging problem. The following theorem (see $[\mathbf{3}]$ ) is perhaps the first result in this direction.

Theorem 1.2. Consider a Lotka-Volterra system (1) restricted to the flow invariant set $\mathbb{R}_{+}^{n} \equiv\left\{\left(x_{1}, \ldots, x_{n}\right) \in \mathbb{R}^{n}: x_{j}>0, j=1, \ldots, n\right\}$, and assume that (i) the system has a singular point, and (ii) is stably dissipative. Then there exists a global attractor and the dynamics on the attractor are Hamiltonian.

By "stably dissipative" we mean that the system is dissipative and every system close to it is also dissipative. As we mentioned before, the notion of dissipative system is due to Volterra. Stably dissipative systems where first studied by Redheffer and collaborators (see $[\mathbf{2 1}, \mathbf{2 2}$, $\mathbf{2 3}, \mathbf{2 4}, \mathbf{2 5}$ ]) under the name "stably admissible". They gave a beautiful description of the attractor (see Section 4 below) which we will use to prove Theorem 1.2. The hypothesis on the existence of a singular point is equivalent to the assumption that some orbit has a $\alpha$ - or $\omega$-limit point in $\mathbb{R}_{+}^{n}$.

One of Volterra's main goals in introducing these equations was the "mechanization" of biology, and he made quite an effort in trying to pursue this program. While seeking a variational principle for the system, he was successful in finding a Hamiltonian formulation in the case where the interaction matrix is skew-symmetric, at the expense of doubling the number of dimensions (see Section 2 for details). Along the way, a polemic with Levi-Civita arose, an account of which can be found in $[\mathbf{1 0}]$. In this paper we shall give a different solution to the problem of putting system (1) into a Hamiltonian frame. In modern language, our approach is related to Volterra's approach by a reduction procedure. 
This Hamiltonian frame is the basis for the Hamiltonian structure refered to in Theorem 1.2.

Once the Hamiltonian character of the dynamics is established, one would like to understand (i) what type of attractors one can get and (ii) what kind of Hamiltonian dynamics one can have on the attractor. It will follow from our work that this amounts to classify the dynamics of Lotka-Volterra systems with skew-symmetric matrix whose associated graph is a forest. We do not know of such classification but we shall argue that these dynamics can be rather complex.

In the simplest situation, the attractor will consist of the unique fixed point in $\mathbb{R}_{+}^{n}$ and the dynamics will be trivial. It was already observed in [23] that there may exist periodic orbits on (non-trivial) attractors. On the other hand, if the attractor is an integrable Hamiltonian system then one can expect the orbits to be almost periodic. As we will see below, through a detailed study of a 4-dimensional chain of predator-prey systems, non-integrable Hamiltonian system can indeed occur. Therefore, typically, the dynamics of dissipative Lotka-Volterra systems are extremely complex. This is related with a famous conjecture in the theory of Hamiltonian systems which can be stated as follows (see $[\mathbf{2 0 ,} \mathbf{1 7}]$ ):

Typically, dynamics on the common level sets of the Hamiltonian and the Casimirs are ergodic.

This survey is organized in two parts. In the first part we deal with basic notions of general systems of type (1) and prove Theorem 1.2. In the second part we present some examples and also applications of the stably dissipative systems to Volterra's systems of type (2), with and without delays. We finish by mentioning some recent contributions on the subject.

\section{PART I. General Theory}

\section{Basic notions}

Here, we will recall some basic notions and facts concerning general Lotka-Volterra systems which will be useful in the next sections. All of these notions can be traced back to Volterra. For a more detailed account of general properties of Lotka-Volterra systems we refer to the book by Hofbauer and Sigmund [9].

For fixed $d_{j} \neq 0$, the transformation

$$
y_{j}=\frac{1}{d_{j}} x_{j}, \quad j=1, \ldots, n,
$$


takes the Volterra system (1) with interaction matrix $A$, into a new Volterra system with interaction matrix $A D$

$$
\dot{y}_{j}=\varepsilon_{j} y_{j}+\sum_{k=1}^{n} d_{k} a_{j k} y_{j} y_{k}, \quad j=1, \ldots, n .
$$

We can therefore think of (3) as a gauge symmetry of the system. A choice of representative $\left(a_{j k}\right)$ in a class of equivalence under gauge transformations will be called a choice of gauge. Since will often take as phase space $\mathbb{R}_{+}^{n}$, we consider only gauge transformations with $d_{j}>0$ in order to preserve phase space. Note also that the classes of Lotka-Volterra systems introduced in Definition 1.1 above are all gauge invariant.

Many properties of a Lotka-Volterra system can be expressed geometrically in terms of its associated graph $G(A, \varepsilon)$. This is the labeled graph, where with each species $j$ we associate a vertex $\bigcirc$ labeled with $\varepsilon_{j}$ and we draw an edge connecting vertex $j$ to vertex $k$ whenever $a_{j k} \neq 0$.

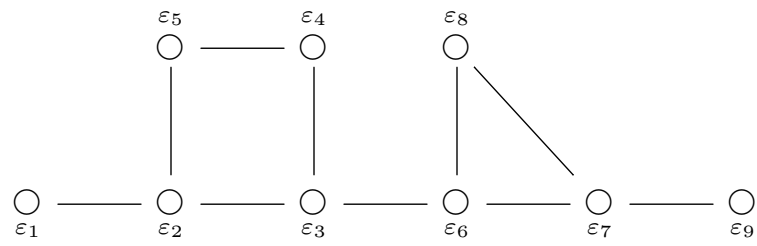

FiguRE 1. Graph $G(A, \varepsilon)$ associated with a system of type (1).

For example, if two systems are gauge equivalent, they have the same unlabeled graph (but not conversely). Also, conservative systems can be caracterized in terms of its graph as it follows from the following proposition also due to Volterra [29, Chapter III, §12]:

Proposition 2.1. A Lotka-Volterra system with interaction matrix $A=$ $\left(a_{j k}\right)$ is conservative if, and only if, $a_{j j}=0$,

$$
a_{j k} \neq 0 \Longrightarrow a_{j k} a_{k j}<0, \quad j \neq k,
$$

and

$$
a_{i_{1} i_{2}} a_{i_{2} i_{3}} \cdots a_{i_{s} i_{1}}=(-1)^{s} a_{i_{s} i_{s-1}} \cdots a_{i_{2} i_{1}} a_{i_{1} i_{s}}
$$

for every finite sequence of integers $\left(i_{1}, \ldots, i_{s}\right)$, with $i_{r} \in\{1, \ldots, n\}$ for $r=1, \ldots, s$. 
In other words a system is conservative if, and only if, (i) the conditions $a_{j j}=0$ and $a_{j k} \neq 0 \Rightarrow a_{k j} \neq 0$ are satisfied and (ii) for each closed path in the diagram with a even (respectively odd) number of vertices the product of the coefficients when we go around in one direction is equal to the product (resp. minus the product) of the coefficients when we go around in the opposite direction. Hence, for example, a system with associated graph as in Figure 1 is conservative if, and only if,

$$
\begin{aligned}
a_{67} a_{78} a_{86} & =-a_{68} a_{87} a_{76}, \\
a_{23} a_{34} a_{45} a_{52} & =a_{25} a_{54} a_{43} a_{32},
\end{aligned}
$$

and moreover the conditions $a_{j j}=0$ and $a_{j k} \neq 0 \Rightarrow a_{j k} a_{k j}<0$ are satisfied.

The most trivial solutions of system (1) are, of course, the fixed points. The fixed points $\mathbf{q}=\left(q_{1}, \ldots, q_{n}\right)$ in $\mathbb{R}_{+}^{n}$ of system (1) are the solutions of the linear system

$$
\varepsilon_{j}+\sum_{k=1}^{n} a_{j k} q_{k}=0, \quad j=1, \ldots, n .
$$

The existence of a fixed point in $\mathbb{R}_{+}^{n}$ is related with the behavior of the orbits in $\mathbb{R}_{+}^{n}$, as it is clear from the following result (see [9, Section 9.2]).

Proposition 2.2. There exists a fixed point $\mathbf{q}=\left(q_{1}, \ldots, q_{n}\right)$ in $\mathbb{R}_{+}^{n}$ of system (1) if, and only if, $\mathbb{R}_{+}^{n}$ contains some $\alpha$-or $\omega$-limit point.

Proof: In one direction the result is clear. On the other hand, assume that there exists no fixed point in $\mathbb{R}_{+}^{n}$ so that for the affine operator $L: \mathbb{R}^{n} \rightarrow \mathbb{R}^{n}$ defined by

$$
L(\mathbf{x})_{j}=\varepsilon_{j}+\sum_{k=1}^{n} a_{j k} x_{k}
$$

one has $0 \notin K=L\left(\mathbb{R}_{+}^{n}\right)$. Then there exists a hyperplane $H$ through the origin disjoint from the convex set $K$, and one can choose $\mathbf{c}=$ $\left(c_{1}, \ldots, c_{n}\right) \in H^{\perp}$ such that

$$
\mathbf{c} \cdot \mathbf{y}>0, \quad \forall \mathbf{y} \in K
$$

Consider now the function $V: \mathbb{R}_{+}^{n} \rightarrow \mathbb{R}$ given by

$$
V(\mathbf{x})=\sum_{j=1}^{n} c_{j} \log \left(x_{j}\right) .
$$

If $x(t)$ is a solution of (1) in $\mathbb{R}_{+}^{n}$ then we compute

$$
\frac{d}{d t} V(\mathbf{x}(t))=\sum_{j=1}^{n} c_{j} \frac{\dot{x}_{j}}{x_{j}}=\mathbf{c} \cdot L(\mathbf{x}(t))>0
$$


where we used (8). Hence, $V$ is a Liapunov function and there can be no $\omega$-limit points since for these one must have $\dot{V}=0$. Similarly, to exclude $\alpha$-limit points one uses the Liapunov function $-V$.

We have just seen that the limit behavior of the orbits is related to the existence of fixed points. On the other hand, the following result shows that the average behavior of the orbits is related to the values of the fixed points (see $[\mathbf{2}])$.

Proposition 2.3. Suppose that $\mathbf{x}(t)$ is an orbit in $\mathbb{R}_{+}^{n}$ of system (1) satisfying $0<m \leq x_{j}(t) \leq M$. Then there is a sequence $\left\{T_{k}\right\}$ such that $T_{k} \rightarrow+\infty$ and a fixed point $\mathbf{q} \in \mathbb{R}_{+}^{n}$ such that

$$
\lim _{k \rightarrow+\infty} \frac{1}{T_{k}} \int_{0}^{T_{k}} \mathbf{x}(t) d t=\mathbf{q}
$$

Moreover, if system (1) has a unique fixed point $\mathbf{q} \in \mathbb{R}_{+}^{n}$ then

$$
\lim _{T \rightarrow+\infty} \frac{1}{T} \int_{0}^{T} \mathbf{x}(t) d t=\mathbf{q}
$$

Proof: Since we have $x_{j}(t) \leq M$, the function

$$
\mathbf{z}(T)=\frac{1}{T} \int_{0}^{T} \mathbf{x}(t) d t
$$

is bounded, and there exists a sequence $\left\{T_{k}\right\}$ such that $T_{k} \rightarrow+\infty$ and the limit

$$
\lim _{k \rightarrow+\infty} \frac{1}{T_{k}} \int_{0}^{T_{k}} \mathbf{x}(t) d t=\mathbf{q}
$$

exists. Since $0<m \leq x_{j}(t)$ it is clear that $\mathbf{q} \in \mathbb{R}_{+}^{n}$. Now, if we integrate (1) along the orbit $\mathbf{x}(t)$ we obtain

$$
\frac{1}{T_{k}}\left(\log \left(x_{j}\left(T_{k}\right)\right)-\log \left(x_{j}(0)\right)\right)=\varepsilon_{j}+\frac{1}{T_{k}} \int_{0}^{T_{k}} \sum_{k=1}^{n} a_{j k} x_{k}(t) d t .
$$

The left-hand side of this equation converges to zero. For the right-hand side we use (12) to compute the limit so we conclude that

$$
0=\varepsilon_{j}+\sum_{k=1}^{n} a_{j k} q_{k}, \quad j=1, \ldots, n,
$$

i.e., $\mathbf{q}$ is a fixed point. 
Now if system (1) has a unique fixed point $\mathbf{q} \in \mathbb{R}_{+}^{n}$ then the linear system (7) has an isolated solution, so the matrix $\left(a_{j k}\right)$ must be nondegenerate. In this case, let us consider any $T \geq 0$ and integrate (1) along the orbit $\mathbf{x}(t)$ from 0 to $T$ :

$$
\frac{1}{T}\left(\log \left(x_{j}(T)\right)-\log \left(x_{j}(0)\right)\right)=\varepsilon_{j}+\frac{1}{T} \int_{0}^{T} \sum_{k=1}^{n} a_{j k} x_{k}(t) d t .
$$

Solving this equation for the averages we obtain

$$
\frac{1}{T} \int_{0}^{T} x_{j}(t) d t=\sum_{k=1}^{n} b_{j k}\left(\frac{1}{T}\left(\log \left(x_{k}(T)\right)-\log \left(x_{k}(0)\right)\right)-\varepsilon_{k}\right),
$$

where $\left(b_{j k}\right)$ is the inverse of $\left(a_{j k}\right)$. By letting $T \rightarrow+\infty$, and using the fact that the $x_{j}(t)$ are bounded, we obtain

$$
\lim _{T \rightarrow+\infty} \frac{1}{T} \int_{0}^{T} x_{j}(t) d t=-\sum_{k=1}^{n} b_{j k} \varepsilon_{k}=q_{j} .
$$

In the case where the interaction matrix $\left(a_{j k}\right)$ is not invertible it is not clear to which fixed point $\mathbf{q}$ does the time average of the orbit converges.

\section{Conservative systems}

In the case were system (1) is conservative Volterra was able to introduce a Hamiltonian structure for the system by doubling the number of variables. We recall now Volterra's construction, so we assume that system (1) is conservative and a choice of gauge has been made so that the matrix $\left(a_{j k}\right)$ is skew-symmetric. Volterra introduces new variables $Q_{j}$ (which he calls quantity of life) through the formula ${ }^{1}$ :

$$
Q_{j}=\int_{0}^{t} x_{j}(\tau) d \tau, \quad j=1, \ldots, n,
$$

and rewrites system (1) as a second order ordinary differential equation (ODE):

$$
\ddot{Q}_{j}=\varepsilon_{j} \dot{Q}_{j}+\sum_{k=1}^{n} a_{j k} \dot{Q}_{j} \dot{Q}_{k}, \quad j=1, \ldots, n .
$$

Then he observes that the function $H=\sum_{j=1}^{n}\left(\varepsilon_{j} Q_{j}-\dot{Q}_{j}\right)$ is a first integral of the system because, on account of skew-symmetry, one has

$$
\dot{H}=-\sum_{j, k=1}^{n} a_{j k} \dot{Q}_{j} \dot{Q}_{k}=0 \text {. }
$$

\footnotetext{
${ }^{1}$ One might argue about the "definition" of the $Q_{j}$ 's. The full justification of this procedure will be given later in the section.
} 
Now, if one introduces another set of variables $P_{j}$ by the formula

$$
P_{j}=\log \dot{Q}_{j}-\frac{1}{2} \sum_{k=1}^{n} a_{j k} Q_{k}, \quad j=1, \ldots, n,
$$

(which are well defined when we restrict the original system to $\mathbb{R}_{+}^{n}$ ), then, in the coordinates $\left(Q_{j}, P_{j}\right)$, the function $H$ is expressed as

$$
H=\sum_{j=1}^{n} \varepsilon_{j} Q_{j}-\sum_{j=1}^{n} e^{\left(P_{j}+\frac{1}{2} \sum_{k=1}^{n} a_{j k} Q_{k}\right)} .
$$

A simple computation shows that system (16) can be rewritten in the following Hamiltonian form

$$
\left\{\begin{array}{l}
\dot{P}_{j}=\frac{\partial H}{\partial Q_{j}}, \\
\dot{Q}_{j}=-\frac{\partial H}{\partial P_{j}},
\end{array} \quad j=1, \ldots, n .\right.
$$

We shall now reverse the all procedure and reformulate it in the language of Poisson manifolds ${ }^{2}$. Recall that the modern approach to Hamiltonian systems is based on the following generalization of the notion of a Poisson bracket (see for example [1], [17]).

Definition 3.1. A POISSON BRACKET on a smooth manifold $M$ is a bilinear operation $\{\}:, C^{\infty}(M) \times C^{\infty}(M) \rightarrow C^{\infty}(M)$ on the space of smooth functions satisfying the following properties:

i) $\left\{f_{1}, f_{2}\right\}=-\left\{f_{2}, f_{1}\right\}$ (skew-symmetry);

ii) $\left\{f_{1} f_{2}, f\right\}=f_{1}\left\{f_{2}, f\right\}+\left\{f_{1}, f\right\} f_{2}$ (Leibnitz's identity);

iii) $\left\{f_{1},\left\{f_{2}, f_{3}\right\}\right\}+\left\{f_{2},\left\{f_{3}, f_{1}\right\}\right\}+\left\{f_{3},\left\{f_{1}, f_{2}\right\}\right\}=0$ (Jacobi's identity).

A Hamiltonian system on a Poisson manifold $M$ is defined by a choice of a function $h \in C^{\infty}(M)$, namely, the defining equations for the flow are

$$
\dot{x}=X_{h}(x),
$$

where the Hamiltonian vector field $X_{h}$ is the vector field on $M$ defined by

$$
X_{h}(f)=\{f, h\}, \quad \forall f \in C^{\infty}(M) .
$$

\footnotetext{
${ }^{2}$ One needs here the more general concept of Poisson manifold rather than symplectic manifold since, as we shall see shortly, the Poisson bracket associated with the original system is, in general, degenerate.
} 
For system (19) $M=\mathbb{R}^{2 n}$ and the Poisson bracket in question is, of course, the classical Poisson bracket associated with the standard symplectic structure $\omega_{s}=\sum_{j=1}^{n} d Q_{j} \wedge d P_{j}$ :

$$
\left\{f_{1}, f_{2}\right\}_{s}=\sum_{j=1}^{n}\left(\frac{\partial f_{1}}{\partial P_{j}} \frac{\partial f_{2}}{\partial Q_{j}}-\frac{\partial f_{2}}{\partial P_{j}} \frac{\partial f_{1}}{\partial Q_{j}}\right)
$$

When we take the function $H$ given by (18) as the Hamiltonian function, it is clear that system (19) takes the canonical form

$$
\dot{x}_{i}=\left\{x_{i}, H\right\}_{s}, \quad i=1, \ldots, 2 n .
$$

The key remark to reverse Volterra's procedure is the following: system (19) has $n$, time-dependent (if $\varepsilon_{j} \neq 0$ ), first integrals given by the formulas

$$
I_{j}\left(Q_{j}, P_{j}, t\right)=P_{j}-\frac{1}{2} \sum_{k=1}^{n} a_{j k} Q_{k}-\varepsilon_{j} t, \quad j=1, \ldots, n .
$$

In fact, one checks easily that

$$
\frac{\partial I_{j}}{\partial t}+\left\{I_{j}, H\right\}_{s}=0
$$

Moreover, the first integrals $I_{j}$ satisfy the following relation

$$
\left\{I_{j}, I_{k}\right\}_{s}=a_{j k}
$$

A standard result (see [17]) in the theory of Hamiltonian systems says that a family of $r$-independent, Poisson commuting integrals, allows one to reduce the dimension of the system by $2 r$. Hence, if the $n$ integrals $I_{j}$ had vanishing Poisson bracket, we would be able to reduce the dimension of the system by $2 n$, and the equations would be integrable by quadratures. Condition (23) of course does not give such a complete integrability, but it is enough to guarantee that the corresponding Hamiltonian vector fields commute

$$
\left[X_{I_{j}}, X_{I_{k}}\right]=0, \quad j, k=1, \ldots, n
$$

This allow us to perform a standard (non-Hamiltonian) symmetry reduction and reduce the dimension of the system by $n$. 
Theorem 3.2. The map $\Psi:\left(Q_{i}, P_{i}\right) \mapsto x_{j}$ defined by

$$
x_{j}=e^{\left(P_{j}+\frac{1}{2} \sum_{k=1}^{n} a_{j k} Q_{k}\right)}, \quad \forall(Q, P) \in \mathbb{R}^{2 n}
$$

is a Poisson map from $\mathbb{R}^{2 n}$ with the canonical Poisson bracket (21) to $\mathbb{R}_{+}^{n}$ with bracket

$$
\left\{f_{1}, f_{2}\right\}=\sum_{j<k} a_{j k} x_{j} x_{k}\left(\frac{\partial f_{1}}{\partial x_{j}} \frac{\partial f_{2}}{\partial x_{k}}-\frac{\partial f_{2}}{\partial x_{j}} \frac{\partial f_{1}}{\partial x_{k}}\right) .
$$

If $\left(q_{1}, \ldots, q_{n}\right) \in \mathbb{R}_{+}^{n}$ is a fixed point of $(1)$, this map reduces the enlarged system (19) to the Volterra system (1).

Proof: One readily verifies that (25) satisfies the conditions of the Definition 3.1. It is also a routine calculation to check that the map $\Psi:\left(Q_{i}, P_{i}\right) \mapsto x_{j}$ satisfies

$$
\{f \circ \Psi, g \circ \Psi\}_{s}=\{f, g\} \circ \Psi, \quad \forall f, g \in C^{\infty}\left(\mathbb{R}_{+}^{n}\right) .
$$

If there is an equilibrium and we let

$$
h=\sum_{j=1}^{n}\left(x_{j}-q_{j} \log x_{j}\right),
$$

we check that $H=h \circ \Psi$, and that system (1) can be written in the form

$$
\dot{x}_{j}=\left\{x_{j}, h\right\}, \quad j=1, \ldots, n .
$$

Hence $\Psi$ reduces the enlarged system (19) to the Volterra system (1).

We leave it to the reader to check that if one considers the action on $\mathbb{R}^{2 n}$ of the (abelian) group of symmetries $G$ generated by the Hamiltonian vector fields $X_{I_{j}}$, then the map $\Psi: \mathbb{R}^{2 n} \rightarrow \mathbb{R}_{+}^{n}$ is exactly the quotient map $\mathbb{R}^{2 n} \rightarrow \mathbb{R}^{2 n} / G$. Therefore the reduction given in Theorem 3.2 is in fact a symmetry reduction.

Remarks.

(i) In general, one cannot get way without some assumption of the type of (7) and so it is not possible to give a Hamiltonian formulation without introducing new variables (if, for example, $\left(a_{j k}\right)=0$ and $\varepsilon_{j}>0$ then the origin is a source and system (1) cannot be Hamiltonian).

(ii) In [19] the Hamiltonian structure (25) is also introduced, along with other Hamiltonian formulations valid for particular classes of interaction matrices. However, there is no reference to its relation to the Volterra Hamiltonian formulation. 
When we combine these ideas with Volterra's criteria for a system to be conservative we obtain

Corollary 3.3. Assume system (1) has a fixed point in $\mathbb{R}_{+}^{n}$. If the matrix associated with the system satisfies $a_{j j}=0$,

$$
a_{j k} \neq 0 \Longrightarrow a_{j k} a_{k j}<0
$$

and the graph is a forest, then the system has a direct Hamiltonian formulation.

Remark. If we do not allow the sign change in condition (6) then we obtain a necessary and sufficient condition for the matrix to be symmetrizable. In this case, the system is gradient with respect to the "metric" $d s^{2}=\sum_{j k}\left(d_{j} a_{j k} x_{j} x_{k}\right) d x_{j} d x_{k}($ see $[\mathbf{1 3}])$.

\section{Dissipative systems}

We now turn to the study of dissipative systems. Since we want our results to persist under small perturbation we introduce the following definition.

Definition 4.1. A Perturbation of a Lotka-Volterra system with interaction matrix $A$ is any Lotka-Volterra system with interaction matrix $\tilde{A}$ such that

$$
\tilde{a}_{j k}=0 \Leftrightarrow a_{j k}=0 .
$$

A Lotka-Volterra system with interaction matrix $A$ is called STABLY DISSIPATIVE if every sufficiently small perturbation is dissipative:

$$
\exists \delta>0: \max _{j k}\left|a_{j k}-\tilde{a}_{j k}\right|<\delta \Longrightarrow \tilde{A} \text { is dissipative. }
$$

Note that we only allow perturbations that have the same graph as the original system. The notion of stably dissipative system is due to Redheffer $e t$ al. who in a series of papers $[\mathbf{2 1}, \mathbf{2 2}, \mathbf{2 3}, \mathbf{2 4}, \mathbf{2 5}]$ have studied the asymptotic stability of this class of systems. Also they use instead the name stably admissible. Since what they call admissible is called by Volterra dissipative [29, Chapter III], we prefer the term stably dissipative. For conditions for a matrix to be stably dissipative we refer to [22].

Let us start then with a stably dissipative Lotka-Volterra system having a fixed point $\mathbf{q}=\left(q_{1}, \ldots, q_{n}\right) \in \mathbb{R}_{+}^{n}$ :

$$
\left\{\begin{array}{l}
\dot{x}_{j}=\varepsilon_{j} x_{j}+\sum_{k=1}^{n} a_{j k} x_{j} x_{k}, \\
\varepsilon_{j}+\sum_{k=1}^{n} a_{j k} q_{k}=0,
\end{array} \quad j=1, \ldots, n .\right.
$$


The system is dissipative so we can choose a diagonal matrix $D>0$ such that $A D \leq 0$. For stably dissipative systems this choice can be improved [24]:

Lemma 4.2. One can choose a positive matrix $D=\operatorname{diag}\left(d_{1}, \ldots, d_{n}\right)$ such that $A D \leq 0$ and the following condition holds

$$
\sum_{j, k=1}^{n} d_{k} a_{j k} w_{i} w_{j}=0 \Longrightarrow a_{j j} w_{j}=0, \quad j=1, \ldots, n .
$$

Proof: Given $A=\left(a_{i j}\right)$ such that the associated system is stably dissipative we consider the perturbation $\tilde{A}=\left(\tilde{a}_{j k}\right)$ given by

$$
\tilde{a}_{j k}=a_{j k}, \quad j \neq k, \quad \tilde{a}_{j j}=(1-\delta) a_{j j} .
$$

Also, choose $D>0$ such that $\tilde{A} D \leq 0$. Since $a_{j j} \leq 0$ and

$$
\sum_{j, k=1}^{n} d_{k} a_{j k} w_{i} w_{j}=\sum_{j, k=1}^{n} d_{k} \tilde{a}_{j k} w_{j} w_{k}+\delta \sum_{j=1}^{n} d_{j} a_{j j} w_{j}^{2},
$$

we see that $A D \leq 0$ and

$$
\sum_{j, k=1}^{n} d_{k} a_{j k} w_{i} w_{j}=0 \Longrightarrow a_{j j} w_{j}=0, \quad j=1, \ldots, n .
$$

If $D=\operatorname{diag}\left(d_{1}, \ldots, d_{n}\right)$ is a matrix as in the previous lemma, we perform the change of gauge $x_{j} \mapsto \frac{1}{d_{j}} x_{j}$ so we can assume that $A \leq 0$ and

$$
\sum_{j, k=1}^{n} a_{j k} w_{i} w_{j}=0 \Longrightarrow a_{j j} w_{j}=0, \quad j=1, \ldots, n .
$$

Then we have a Liapunov function given by

$$
V=\sum_{j=1}^{n}\left(x_{j}-q_{j} \log x_{j}\right) .
$$

In fact, we find that

$$
\dot{V}=\sum_{j, k=1}^{n} a_{j k}\left(x_{j}-q_{j}\right)\left(x_{k}-q_{k}\right) \leq 0 .
$$

By LaSalle's Theorem [11], the solutions exist for all $t \geq 0$ and the set $\dot{V}=0$ contains an attractor. Therefore one would like to understand the set $\dot{V}=0$. 
We shall now recall Redheffer's beautiful description of the attractor in terms of the reduced graph of the system. Notice that by (28), (29) and (30) solutions on the set $\dot{V}=0$ satisfy

$$
\left\{\begin{array}{l}
\dot{x}_{j}=x_{j} \sum_{k=1}^{n} a_{j k}\left(x_{k}-q_{k}\right), \quad j=1, \ldots, n . \\
a_{j j}\left(x_{j}-q_{j}\right)=0,
\end{array}\right.
$$

Therefore, one has either $a_{j j}=0$ or $a_{j j}<0$, and in the later case we have $x_{j}=q_{j}$ on the attractor.

It will be convenient to modify slightly the notion of graph associated with the system we introduced above. One now draws a black dot at vertex $j$ if either $a_{j j}<0$, or $a_{j j}=0$ and somehow we have shown that $x_{j}=q_{j}$ on the attractor. Otherwise, one draws an open circle $\bigcirc$ at vertex $j$. It is also convenient to put a $\oplus$ at vertex $j$ if one can show that $x_{j}$ is constant on the attractor (an intermediate stage between black dots and open circles).

We have $[\mathbf{2 3}]$ :

Lemma 4.3. The following propagation rules are valid:

(a) If there is a $\bullet$ or $\oplus$ at vertex $j$ and $\bullet$ at all neighbors of $j$ except one vertex $l$, then we can put a $\bullet$ at vertex $l$.

(b) If there is $a \bullet$ or $\oplus$ at vertex $j$, and $a \bullet$ or $\oplus$ at all neighbors of $j$ except one vertex $l$, then we can put $a \oplus$ at vertex $l$.

(c) If there is $\bigcirc$ at vertex $j$, and $\bullet$ or $\oplus$ at all neighbors of $j$, then we can put $\oplus$ at vertex $j$.

Proof: The proof is a straightforward application of (31).

One calls the reduced graph $R(A)$ of the system, the graph obtained by repeated use of the rules of reduction (a), (b) and (c). Figure 2 gives an example of a graph and its reduced graph obtained by successive application of these rules. For more on the reduced graph we refer to [23]. Here we shall only need the following fact which follows from the results in $[\mathbf{2 4}]$.
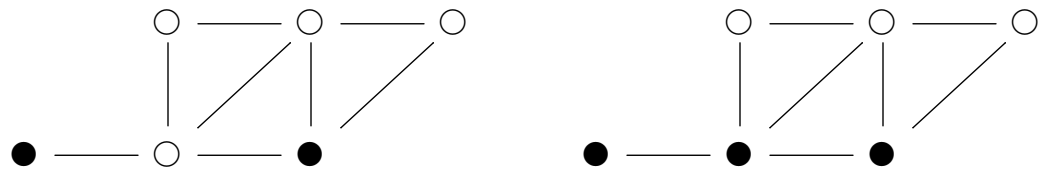

Figure 2. A graph $G(A)$ and its reduced form $R(A)$. 
Proposition 4.4. Let $K$ denote the subgraph of the reduced graph of a stably dissipative Lotka-Volterra system formed by vertices with or $\oplus$ and connections between them. Then $K$ is a forest, i.e., $K=$ $K_{1} \cup \cdots \cup K_{r}$ (disjoint) where each $K_{i}$ is a tree.

Proof: We have to rule out the existence of a closed path whose vertices are all of type $\bigcirc$ or $\oplus$. Assume we had such a closed path and label its vertices from 1 to $m$. Then one has $a_{j j}=0$ for each $1 \leq j \leq m$, so given two adjacent vertices $j$ and $k$ in this closed closed path we must have

$$
a_{j k}+a_{k j}=0
$$

on account of the condition $A \leq 0$. In other words the reduced system whose graph is the closed path is conservative. By Proposition 2.1, this can happen if, and only if

$$
a_{12} \cdots a_{m-1 m} a_{m 1}=(-1)^{m} a_{1 m} a_{m m-1} \cdots a_{21} .
$$

Clearly, this condition cannot hold for all small perturbations. Hence the original system would not be stably dissipative.

We are now in condition to prove Theorem 1.2 which we state as follows:

Theorem 4.5. Consider a Lotka-Volterra system (1) restricted to the flow invariant set $\mathbb{R}_{+}^{n} \equiv\left\{\left(x_{1}, \ldots, x_{n}\right) \in \mathbb{R}^{n}: x_{j}>0, j=1, \ldots, n\right\}$, and assume that (i) the system has a singular point $\mathbf{q} \in \mathbb{R}_{+}^{n}$, and (ii) is stably dissipative. Then the dynamics on the set $\dot{V}=0$ are Hamiltonian. Moreover, they can be described by a Lotka-Volterra system of dimension $m \leq n$.

Proof: Consider the system restricted to $\dot{V}=0$. We split the variables $x_{j}$ into two groups labeled by sets $J_{\circ}$ and $J_{\bullet}$. In the first group $\left\{x_{j}\right\}_{j \in J_{\circ}}$ we have all the $x_{j}$ 's corresponding to vertices with open circles $\bigcirc$ or $\oplus$ in $R(A)$, while the second group $\left\{x_{j}\right\}_{j \in J_{\bullet}}$ we have all the $x_{j}$ 's corresponding to vertices with black circles $\bigcirc R(A)$. For $j \in J_{\text {• we have }}$ $x_{j}=q_{j}$, hence the restricted system satisfies

$$
\begin{cases}\dot{x}_{j}=\left(\varepsilon_{j}+\sum_{k \in J_{\bullet}} a_{j k} q_{k}\right) x_{j}+\sum_{k \in J_{\circ}} a_{j k} x_{j} x_{k} & \text { if } j \in J_{\circ}, \\ x_{j}=q_{j} & \text { if } j \in J_{\bullet} .\end{cases}
$$

Therefore if we define $\tilde{\varepsilon}_{j}=\varepsilon_{j}+\sum_{k \in J_{\bullet}} a_{j k} q_{k}, \tilde{a}_{j k}=a_{j k}\left(j, k \in J_{\circ}\right)$, we obtain a new Volterra type system:

$$
\dot{x}_{j}=\tilde{\varepsilon}_{j} x_{j}+\sum_{k \in J_{\circ}} \tilde{a}_{j k} x_{j} x_{k}, \quad j \in J_{\circ},
$$


where the graph associated with the matrix $\tilde{A}=\left(\tilde{a}_{j k}\right)_{j, k \in J_{\circ}}$ is precisely the subgraph $K$ of the reduced graph $R(A)$ formed by vertices with $\bigcirc$ or $\oplus$ and connections between them. Note that this matrix satisfies $\tilde{a}_{j j}=0$, and that there exists a diagonal matrix $D>0$ such that $\tilde{A} D \leq 0$. But this implies

$$
d_{j} \tilde{a}_{j k}+d_{k} \tilde{a}_{k j}=0
$$

which shows that

$$
a_{j k} \neq 0 \Longrightarrow a_{j k} a_{k j}<0 .
$$

Note also that the $\left(q_{j}\right)_{j \in J}$ form a solution of the system

$$
\tilde{\varepsilon}_{j}+\sum_{k \in J_{\circ}} \tilde{a}_{j k} q_{k}=0, \quad j \in J_{\circ} .
$$

By Proposition 4.4, we are in the conditions of Corollary 3.3, so system (33) has a Hamiltonian formulation.

The proof shows that the dynamics on the attractor can be described by a Lotka-Volterra system of dimension $m \leq n$ whose associated graph is a tree, which is conservative and has a fixed point in $\mathbb{R}_{+}^{m}$. Conversely, any such system describes an attractor, since any system whose associated graph is a tree is stably dissipative.

\section{PART II. ExAmples AND APplicAtions}

\section{Examples}

Example 5.1. In [3, p. 159, eq. (33)], the authors give a detailed analysis of a 4-dimensional Lotka-Volterra system in order to illustrate the complexity of the dynamics that can occur on the attractor. The system is the following:

$$
\left\{\begin{array}{l}
\dot{x}_{1}=-x_{1}+x_{1} x_{2}, \\
\dot{x}_{2}=+x_{2}-x_{2}\left(x_{1}-\delta x_{3}\right), \\
\dot{x}_{3}=-x_{3}+x_{3}\left(x_{4}-\delta x_{2}\right), \\
\dot{x}_{4}=+x_{4}-x_{4} x_{3} .
\end{array}\right.
$$

They have included a parameter $\delta$ which must be restricted to ] $-1,+\infty[$ since they need the fixed point $\mathbf{q}=(1+\delta, 1,1,1+\delta)$ to belong to $\mathbb{R}_{+}^{4}$. The interaction matrix is skew-symmetric:

$$
\left(a_{i j}\right)=\left(\begin{array}{cccc}
0 & 1 & 0 & 0 \\
-1 & 0 & \delta & 0 \\
0 & -\delta & 0 & 1 \\
0 & 0 & -1 & 0
\end{array}\right)
$$


This is an extremely interesting system for which they show, among other properties, that

- the system is non-integrable in the sense of Arnol'd-Liouville;

- the dynamics of the system is equivalent to the dynamics of a homeomorphism of a sphere;

- the system has families of periodic orbits whose stability is determined by an associated Sturm-Liouville problem;

- one can find regions in the space of parameters where periodic orbits are strongly hyperbolic.

We believe that both this 4-dimensional system and higher dimension generalizations deserve further study, and can help understanding the conjecture stated in the end of the Introduction.

Example 5.2. In $[\mathbf{1 6}]$, it is considered the Volterra system of retarded type defined by equations (2) with the constant $r$ satisfying $0<r<\infty$ :

$$
\dot{N}_{i}(t)=N_{i}(t)\left[\varepsilon_{i}+\sum_{s=1}^{n} p_{i s} N_{s}(t)+\sum_{s=1}^{n} \int_{-r}^{0} N_{s}(t+\theta) F_{i s}(-\theta) d \theta\right],
$$

where $\varepsilon_{i}, p_{i s}$ are constants, $N_{i}=N_{i}(t)>0$, for $i, s=1, \ldots, n$, and $F_{i s}(-\theta)$ are the values at $-\theta$ of the real memory functions $F_{i s}$ assumed to be continuous on the interval $[0, r]$; here also $i=1,2, \ldots, n$. As an example of memory functions it is usual consider $F_{i s}(\tau)=c_{i s} \exp \left(-\kappa_{i s} \tau\right)$, where $c_{i s}$ are real numbers, $\kappa_{i s} \geq 0$ and $\tau \in[0, r]$.

Using a special "cut off" technique for that RFDE combined with a classical Kurzweil's result, the authors were able to describe a set of global bounded solutions of system (36) (see Theorem 4.1 of [16]) as solutions of a suitable system of ODEs.

The authors of [16] also analyzed a special case of system (36) with $n=2$ species and constant memory functions; with the knowledge of the structure of the well known 2-dimensional prey-predator Lotka-Volterra model they exhibited a compact invariant set for the RFDEs.

We saw in the previous Example 5.1, that complex dynamics can occur in Lotka-Volterra systems of ODEs when $n \geq 4$. This fact and the mentioned Theorem 4.1 of [16] imply that some systems of type (36) with $n \geq 4$ may have very complicated behavior.

Example 5.3. This example shows that the authors in [15] decided to keep themselves with a Volterra RFDEs of the type (36) in the case of $n=3$ species because is still possible to give a geometric description of a set of global bounded solutions, due, mainly, to the Poisson integrability (see [3]) of two associated 3-dimensional Lotka-Volterra ODE systems. 
At this point we also mention that another important tool used here is the main result of the previous example, that is, Theorem 4.1 of $[\mathbf{1 6}]$.

Thus, let us consider the following Volterra RFDEs in $R_{+}^{3}$ :

$$
\left\{\begin{array}{l}
\dot{N}_{1}=N_{1}\left[-\varepsilon_{1}+a N_{2}+a N_{3}+\delta \int_{-r}^{0} N_{1}(t+\theta) d \theta\right], \\
\dot{N}_{2}=N_{2}\left[+\varepsilon_{2}-a N_{1}-a N_{3}+\delta \int_{-r}^{0} N_{2}(t+\theta) d \theta\right] \\
\dot{N}_{3}=N_{3}\left[+\varepsilon_{3}-a N_{1}+a N_{2}+\delta \int_{-r}^{0} N_{3}(t+\theta) d \theta\right],
\end{array}\right.
$$

where the given constants satisfy

$$
r>0, \quad a>0, \quad \varepsilon_{1}>0, \quad \varepsilon_{2}>0, \quad \varepsilon_{3}=\varepsilon_{2}-\varepsilon_{1}>0
$$

and

$$
\varepsilon_{1}<\varepsilon_{2}<2 \varepsilon_{1} .
$$

Here $\delta$ is a small parameter such that

$$
\frac{0<\delta<a\left(2 \varepsilon_{1}-\varepsilon_{2}\right)}{r \varepsilon_{2}} .
$$

The invariant set to be described is a special set $\mathcal{K}$ of global bounded solutions of system (37) with conditions (38), (39) and (40). $\mathcal{K}$ is a subset of the phase space $\mathcal{C}=C^{0}\left([-r, 0], \mathbb{R}^{3}\right)$ which is a "solid cylinder" homeomorphic, under the map $\rho: \phi \in \mathcal{C} \mapsto \rho(\phi)=\phi(0) \in \mathbb{R}^{3}$, to a solid cylinder contained in $\mathbb{R}^{3}$.

The boundary $\partial \mathcal{K}$ of $\mathcal{K}$ is the union of a closed 2-dimensional cilin$\operatorname{der} \mathcal{C}(\mathcal{K})$ with two open disks, the two "bases" of $\mathcal{K}$, each one of them being invariant under $(37)$; the cylinder $\mathcal{C}(\mathcal{K})$ is the union of a set of $r$-periodic orbits of $(37)$ contained in $\mathbb{R}^{3}(\mathcal{C}(\mathcal{K})$ is in fact the union of a one-parameter family of orbits). The geometry of the sets $\mathcal{K}$ and $\partial \mathcal{K}$ will be clarified along the proof of the main Theorem 1.1 of [15], that can be stated in the following way:

Theorem. Let $r>2 \pi\left[\varepsilon_{1}\left(\varepsilon_{2}-\varepsilon_{1}\right)\right]^{-1 / 2}$. Then there is a $\delta_{0}>0$ such that for all $\delta \in\left(0, \delta_{0}\right]$ system $(37)$ has in $C=C^{0}\left([-r, 0], \mathbb{R}_{+}^{3}\right)$ a 3 -dimensional compact invariant topological manifold $\mathcal{K}$. This manifold is homeomorphic to a solid cylinder in $\mathbb{R}_{+}^{3}$ under the map $\rho: \phi \in \mathcal{C} \rightarrow$ $\phi(0) \in \mathbb{R}^{3}$. The set $\partial \mathcal{K}$ is the disjoint union of two planar open disks with a bi-dimensional cylinder $\mathcal{C}(\mathcal{K})$ which is the union of a continuous one-parameter family of $r$-periodic orbits of (37). Moreover, any other $r$-periodic orbit of (37) is contained in $\mathcal{K}$ and the flow of (37) restricted to $\mathcal{K}$ is the flow of a $C^{1}$ vector-field. 
As one can see in [15], it is possible to show that the $r$-periodic solutions of (37) inside $\partial \mathcal{K}$ are also solutions of an auxiliary 3-dimensional Lotka-Volterra system. The search of solutions of $(37)$ in $\mathbb{R}_{+}^{3}$ is reduced to the integrability of a one-parameter family of planar ODE systems, each of them corresponding to a vector-field evolving in a surface transversal to the line of equilibria of the auxiliary Lotka-Volterra system. Each surface is the level of a Casimir integral and the corresponding planar ODE is in fact Hamiltonian. For the (quite long) proof of that theorem we need to introduce a series of lemmas and remarks (see [15, pp. 261-282]) that allow us to use Theorem 4.1 of [16].

Example 5.4. In this example we will see an application of stably dissipative Lotka-Volterra equations to a special system of RFDEs with a property called reducibility. A RFDEs is said to be reducible if its global bounded solutions are solutions of a system of ordinary differential equations.

The RFDEs we will deal with is a Volterra system with infinite delay of the following form

$$
\dot{x}_{j}(t)=x_{j}(t)\left[\varepsilon_{j}+\sum_{k=1}^{m} a_{j k} x_{k}(t)+\sum_{k=1}^{m} \int_{-\infty}^{0} x_{k}(t+\theta) F_{j k}(\theta) d \theta\right],
$$

where $\varepsilon_{j}, a_{j k}$ are constants and $x_{j}=x_{j}(t)>0$, for $j, k=1, \ldots, m$, and $F_{j k}(\theta)$ are the memory functions.

Equation (41) is a functional differential equation, so in standard notation this equation has the form $\dot{x}(t)=f\left(x_{t}\right)$, where $x_{t}(\theta)=x(t+\theta)$ for $\theta \in(-\infty, 0]$ and, in an appropriate phase space, it generates a nonlinear dynamical system $([\mathbf{5}],[\mathbf{6}])$.

A phase space for the equation (41) is an open subset of the following separable Banach space $C_{\gamma}, \gamma>0$, defined in the following way:

$$
C_{\gamma}=\left\{\varphi \in C\left((-\infty, 0], \mathbb{R}^{m}\right): \lim _{\theta \rightarrow-\infty} e^{\gamma \theta} \varphi(\theta) \text { exists }\right\}
$$

(see $[6])$. Since $\mathbb{R}_{+}^{m}$ is open in $\mathbb{R}^{m}$, the phase space for (41) is the set $C_{\gamma}^{+}$, open in $C_{\gamma}$, that is, their elements are continuous paths $\varphi \in$ $C\left((-\infty, 0], \mathbb{R}_{+}^{m}\right)$.

The space $C_{\gamma}$ is isometrically isomorphic to the Banach space $C^{0}=$ $C\left([-r, 0], \mathbb{R}^{m}\right), 0<r<\infty$, where $C^{0}$ is endowed with the sup norm and $C_{\gamma}$ with the norm

$$
\|\varphi\|_{\gamma}=\sup _{\theta \leq 0}\left\{e^{\gamma \theta}|\varphi(\theta)|\right\}
$$


The isometry $\mathcal{I}_{\gamma}$ is given by the following map: for a given function $\varphi \in C_{\gamma}$ we define $u=\mathcal{I}_{\gamma}(\varphi) \in C^{0}$ by

$$
u(s)=\left[\mathcal{I}_{\gamma}(\varphi)\right](s):= \begin{cases}e^{\frac{\gamma s}{r+s}} \varphi\left(\frac{\gamma s}{r+s}\right) & \text { if } s \in(-r, 0], \\ \lim _{\theta \rightarrow-\infty} e^{\gamma \theta} \varphi(\theta) & \text { if } s=-r .\end{cases}
$$

To define the inverse $\mathcal{I}_{\gamma}^{-1}$, one considers $u:[-r, 0] \rightarrow \mathbb{R}^{m}$ and associates it to the function $\mathcal{I}_{\gamma}^{-1}(u)=\varphi \in C_{\gamma}$, where $\varphi:(-\infty, 0] \rightarrow \mathbb{R}^{m}$ is given by

$$
\varphi(\theta)=\left[\mathcal{I}_{\gamma}^{-1}(u)\right](\theta):=e^{-\gamma \theta} u\left(\frac{r \theta}{1-\theta}\right), \quad \theta \in(-\infty, 0] .
$$

Finally, all the classical results on existence, uniqueness and continuous dependence of solutions for the equations with finite delay also hold in the case of infinite delay with these phase spaces, [6].

If, for simplicity, one chooses in equation (41) the memory functions $F_{j k}=F_{j k}(\theta)$ as

$$
F_{j k}(\theta)=p_{j k} e^{\theta}
$$

one obtains

$$
\dot{x}_{j}=x_{j}\left[\varepsilon_{j}+\sum_{k=1}^{m} a_{j k} x_{k}+\sum_{k=1}^{m} p_{j k} \int_{-\infty}^{0} e^{\theta} x_{k}(t+\theta) d \theta\right],
$$

where $\varepsilon_{j}, a_{j k}$ and $p_{j k}$ are real numbers and $j, k=1, \ldots, m$.

One observes that there exist equilibrium solutions $\mathbf{q}=\left(q_{1}, \ldots, q_{m}\right) \in$ $\mathbb{R}_{+}^{m}$ of (47) if, and only if,

$$
\varepsilon_{j}=-\sum_{k=1}^{m}\left(a_{j k}+p_{j k}\right) q_{k}, \quad j=1, \ldots, m .
$$

The choice (46) for the memory functions will imply the next Theorem 1 which asserts the reducibility of the Volterra system (47) of retarded equations with infinite delay.

One can reduce the Volterra system (47) just by defining the functions

$$
y_{j}(t):=\int_{-\infty}^{0} e^{\theta} x_{j}(t+\theta) d \theta, \quad j=1, \ldots, m,
$$

and obtaining another ODE system

$$
\left\{\begin{array}{l}
\dot{x}_{j}=\varepsilon_{j} x_{j}+\sum_{k=1}^{m} a_{j k} x_{j} x_{k}+\sum_{k=1}^{m} p_{j k} x_{j} y_{k}, \\
\dot{y}_{j}=x_{j}-y_{j} .
\end{array}\right.
$$


In the following, we let $0<\gamma<1$ and take $C_{\gamma}^{+}$for the phase space of (47). Equations (48) are related to system (47) in the following way:

Theorem 1. Any global solution $x_{j}=x_{j}(t)$ of the Volterra system (47) defines a global solution $\left(x_{j}, y_{j}\right)=\left(x_{j}(t), y_{j}(t)\right)$ of the ODE system (48). Moreover, this solution satisfies the following condition

$$
\lim _{\theta \rightarrow-\infty} e^{\theta} y_{j}(\theta)=0, \quad j=1, \ldots, m .
$$

Conversely, if $\left(x_{j}, y_{j}\right)=\left(x_{j}(t), y_{j}(t)\right)$ is a global solution of the ODE system (48) such that (49) is satisfied, then $x_{j}=x_{j}(t)$ is a global solution of (47).

Proof: In fact, from the definition of $y_{j}(t)$ it follows that

$$
e^{\theta} y_{j}(\theta)=\int_{-\infty}^{0} e^{\theta+\tau} x_{j}(\theta+\tau) d \tau=\int_{-\infty}^{\theta} e^{t} x_{j}(t) d t,
$$

so, since $x_{j}=x_{j}(t)$ is a global solution of (47) and $\gamma<1$, we obtain (49). Furthermore, integration by parts yields the second equation in (48).

Conversely, if $\left(x_{j}, y_{j}\right)=\left(x_{j}(t), y_{j}(t)\right)$ is a global solution of the ODE system (48), from the integration of the second equation we obtain

$$
e^{t} y_{j}(t)=e^{t_{0}} y_{j}\left(t_{0}\right)+\int_{t_{0}}^{t} e^{\tau} x_{j}(\tau) d \tau .
$$

Taking the limit $t_{0} \rightarrow-\infty$ and using (49) we obtain

$$
y_{j}(t)=e^{-t} \int_{-\infty}^{t} e^{\tau} x_{j}(\tau) d \tau=\int_{-\infty}^{0} e^{\theta} x_{j}(t+\theta) d \theta,
$$

which implies that $x_{j}=x_{j}(t)$ is a global solution of (47).

This theorem shows that all the information on the global solutions of (48) is useful for the description of the flow of (47).

An immediate consequence of the analysis of (48) is the following:

Proposition 2. The set $\mathbb{R}_{+}^{m} \times \mathbb{R}_{+}^{m}$ is invariant under the flow of the ODE system (48).

Proof: Let $\left(x_{j}(t), y_{j}(t)\right)$ be a solution of (48) with $\left(x_{j}\left(t_{0}\right), y_{j}\left(t_{0}\right)\right) \in$ $\mathbb{R}_{+}^{m} \times \mathbb{R}_{+}^{m}$ and let $I \subset\left[t_{0},+\infty\right)$ denote its maximal interval of existence. Assume that there exists $\tau \in I$ for which either $x_{j}(\tau)=0$ or $y_{j}(\tau)=0$ for some $j=1, \ldots, m$. If $x_{j}(\tau)=0$ then the first equation of (48) implies that $\dot{x}_{j}(\tau)=0$, and so, all the derivatives of $x_{j}(t)$ vanish at $t=\tau$. Since the ODE system (48) is real analytic, we have that $x_{j}(t)=0$ for all $t \in I$, which contradicts the hypothesis $x_{j}\left(t_{0}\right)>0$. 
Therefore, $x_{j}(\tau)>0$ for all $\tau \in I$. If otherwise $y_{j}(\tau)=0$ for some $\tau \in I$, from the second equation of (48) we obtain upon integration

$$
y_{j}(\tau)=e^{t_{0}-\tau} y_{j}\left(t_{0}\right)+\int_{t_{0}}^{\tau} e^{\theta-\tau} x_{j}(\theta) d \theta=0 .
$$

Therefore, we have that

$$
y_{j}\left(t_{0}\right)=-\int_{t_{0}}^{\tau} e^{\theta-t_{0}} x_{j}(\theta) d \theta<0,
$$

contradicting the hypothesis $y_{j}\left(t_{0}\right)>0$. Consequently, we have $y_{j}(\tau)>$ 0 for all $\tau \in I$.

5.1. The relation with the Lotka-Volterra system. Consider again the ODE system

$$
\left\{\begin{array}{l}
\dot{x}_{j}=\varepsilon_{j} x_{j}+\sum_{k=1}^{m} a_{j k} x_{j} x_{k}+\sum_{k=1}^{m} p_{j k} x_{j} y_{k}, \\
\dot{y}_{j}=x_{j}-y_{j}
\end{array}\right.
$$

for $j=1, \ldots, m$, with interation matrix $A=\left(a_{j k}\right)$ and perturbation matrix $P=\left(p_{j k}\right)$. It turns out that, for adequate perturbation matrices, the dynamics of this system is related to the dynamics of the LotkaVolterra equations

$$
\dot{x}_{j}=\varepsilon_{j} x_{j}+\sum_{k=1}^{m} a_{j k} x_{j} x_{k}, \quad j=1, \ldots, m .
$$

These equations were extensively analysed by Duarte, Fernandes and Oliva in [3] where the flow generated by (51) was discussed using a reduction procedure to establish the existence of invariant sets with a Hamiltonian structure.

In the following we use the approach of $[\mathbf{3}]$ to analyse the dynamics of (50).

For a fixed diagonal matrix $D=\operatorname{diag}\left(d_{j}\right)$, with $d_{j}>0, j=1, \ldots, m$, the transformation $X_{j}=x_{j} / d_{j}, Y_{j}=y_{j} / d_{j}$ is a gauge symmetry taking system (50) into

$$
\left\{\begin{array}{l}
\dot{X}_{j}=\varepsilon_{j} X_{j}+\sum_{k=1}^{m} a_{j k} d_{k} X_{j} X_{k}+\sum_{k=1}^{m} p_{j k} d_{k} X_{j} Y_{k}, \\
\dot{Y}_{j}=X_{j}-Y_{j},
\end{array}\right.
$$

which is again a system of the form (50) with interaction matrix $A D$ and perturbation matrix $P D$. A choice of $A=\left(a_{j k}\right)$ and $P=\left(p_{j k}\right)$ in the equivalence class under the above gauge transformation is also called a 
choice of gauge. Notice that, due to the invariance of $\mathbb{R}_{+}^{m} \times \mathbb{R}_{+}^{m}$ given by Proposition 2, the phase space is preserved by the gauge transformation.

Here we discuss the global bounded orbits of (50) and an essential assumption for this discussion is the existence of equilibrium points. There exists an equilibrium solution for $(50), x_{j}=y_{j}=q_{j}$ for $j=$ $1, \ldots, m$, if and only if

$$
\varepsilon_{j}+\sum_{k=1}^{m}\left(a_{j k}+p_{j k}\right) q_{k}=0, \quad j=1, \ldots, m .
$$

In fact, repeating the argument in $[\mathbf{3}]$ we can relate the asymptotic behaviour of the orbits in $\mathbb{R}_{+}^{m} \times \mathbb{R}_{+}^{m}$ with the existence of an equilibrium point of (50), that is a point $(\mathbf{q}, \mathbf{q}) \in \mathbb{R}_{+}^{m} \times \mathbb{R}_{+}^{m}$ such that $\mathbf{q}=\left(q_{1}, \ldots, q_{m}\right)$ satisfies (53).

Proposition 3. There exists a fixed point $(\mathbf{q}, \mathbf{q}) \in \mathbb{R}_{+}^{m} \times \mathbb{R}_{+}^{m}$ of system (50) if and only if $\mathbb{R}_{+}^{m} \times \mathbb{R}_{+}^{m}$ contains some $\alpha$-or $\omega$-limit point.

Proof: Consider the affine operator $L: \mathbb{R}^{m} \times \mathbb{R}^{m} \rightarrow \mathbb{R}^{m} \times \mathbb{R}^{m}$ given by

$$
\begin{aligned}
& L\left(x_{1}, \ldots, x_{m}, y_{1}, \ldots, y_{m}\right)_{j}=\varepsilon_{j}+\sum_{k=1}^{m}\left(a_{j k} x_{k}+p_{j k} y_{k}\right), \\
& L\left(x_{1}, \ldots, x_{m}, y_{1}, \ldots, y_{m}\right)_{m+j}=x_{j}-y_{j},
\end{aligned}
$$

for $j=1, \ldots, m$. If there is no fixed point of $(50)$, then $0 \notin K=$ $L\left(\mathbb{R}_{+}^{m} \times \mathbb{R}_{+}^{m}\right)$. Hence, there exists a hyperplane $H$ through the origin of $\mathbb{R}^{m} \times \mathbb{R}^{m}$ disjoint from the convex set $K$, and we can choose $\mathbf{c}=$ $\left(c_{1}, \ldots, c_{2 m}\right) \in H^{\perp}$ such that $\mathbf{c} \cdot \mathbf{z}>0$ for all $\mathbf{z} \in K$. Then we consider the function $U: \mathbb{R}_{+}^{m} \times \mathbb{R}_{+}^{m} \rightarrow \mathbb{R}$ given by

$$
U\left(x_{1}, \ldots, x_{m}, y_{1}, \ldots, y_{m}\right)=\sum_{j=1}^{m}\left(c_{j} \log x_{j}+c_{m+j} y_{j}\right)
$$

and, if $\mathbf{w}(t)=\left(x_{j}(t), y_{j}(t)\right)$ is a solution of $(50)$, we obtain

$$
\frac{d}{d t} U(\mathbf{w}(t))=\sum_{j=1}^{m}\left(c_{j} L(\mathbf{w}(t))_{j}+c_{m+j} L(\mathbf{w}(t))_{m+j}\right)=\mathbf{c} \cdot L(\mathbf{w}(t))>0 .
$$

This excludes the existence of any $\alpha$ - or $\omega$-limit point, for which one would have $\dot{U}=0$, and concludes the proof.

Under the hypothesis of existence of an equilibrium point, one is able to exhibit a Liapunov function $V$ for (50) by assuming certain conditions for the interaction and perturbation matrices (see [14]). When the interaction matrix $A$ and the perturbation matrix $P$ are both negative 
semidefinite in the sense of quadratic forms, $A \leq 0, P \leq 0$, and $P$ is symmetric, we set

$$
V=\sum_{j=1}^{m}\left(x_{j}-q_{j} \log x_{j}\right)-\frac{1}{2} \sum_{j, k=1}^{m} p_{j k}\left(y_{j}-q_{j}\right)\left(y_{k}-q_{k}\right) .
$$

Computing the derivative of $V$ along the solutions of (50) and using the symmetry of $P$ we obtain

$$
\dot{V}=\sum_{j=1}^{m}\left(x_{j}-q_{j}\right)\left(\varepsilon_{j}+\sum_{k=1}^{m}\left(a_{j k} x_{k}+p_{j k} y_{k}\right)\right)-\sum_{j, k=1}^{m} p_{j k}\left(x_{j}-y_{j}\right)\left(y_{k}-q_{k}\right),
$$

and from (53) we have

$$
\begin{aligned}
\dot{V}=\sum_{j=1}^{m}\left(x_{j}-q_{j}\right)\left(\sum_{k=1}^{m} a_{j k}\left(x_{k}-q_{k}\right)\right. & \left.+p_{j k}\left(y_{k}-q_{k}\right)\right) \\
& -\sum_{j, k=1}^{m} p_{j k}\left(x_{j}-y_{j}\right)\left(y_{k}-q_{k}\right) .
\end{aligned}
$$

Therefore, we conclude

$$
\dot{V}=\sum_{j, k=1}^{m} a_{j k}\left(x_{j}-q_{j}\right)\left(x_{k}-q_{k}\right)+\sum_{j, k=1}^{m} p_{j k}\left(y_{j}-q_{j}\right)\left(y_{k}-q_{k}\right) \leq 0 .
$$

We first consider the case $P<0$ and notice that the level sets of $V$ in a neighbourhood of the equilibrium $(\mathbf{q}, \mathbf{q})$ are $S^{2 m-1}$ spheres. In fact, one easily checks that $(\operatorname{grad} V)_{(\mathbf{q}, \mathbf{q})}=0$ and the Hessian of $V$ at $(\mathbf{q}, \mathbf{q})$ is the positive definite matrix

$$
H(V)_{(\mathbf{q}, \mathbf{q})}=\left[\begin{array}{cc}
\operatorname{diag}\left(q_{1}^{-1}, \ldots, q_{m}^{-1}\right) & 0 \\
0 & -P
\end{array}\right] .
$$

To study the asymptotic behavior of $(x(t), y(t))$ we need to consider the subset of the phase space where $\dot{V}=0$, which by LaSalle's principle contains the $\omega$-limit set of the orbit $(x(t), y(t))$. From (58), on the set $\dot{V}=0$ we have

$$
y_{j}=q_{j}, \quad j=1, \ldots, m,
$$

and, from the second equation in (50) it follows that also

$$
x_{j}=q_{j}, \quad j=1, \ldots, m .
$$

The solution q of (53) is unique since $A \leq 0$ and $P<0$ implies that $A+P<0$ which therefore is nonsingular. Then we conclude that system (50) is dissipative and possesses a global attractor which is a singleton, $\mathcal{A}=\{(\mathbf{q}, \mathbf{q})\}$. Since the solutions $x(t) \in C_{\gamma}^{+}, t \geq 0$, of the Volterra 
equations (47) determine solutions $\left(x_{j}(t), y_{j}(t)\right) \in \mathbb{R}_{+}^{m} \times \mathbb{R}_{+}^{m}, t \geq 0$, we obtain the following

Proposition 4. If (53) has a solution $\mathbf{q} \in \mathbb{R}_{+}^{m}$, the interaction matrix $A$ is negative semidefinite, $A \leq 0$, and the perturbation matrix $P$ is symmetric negative definite, $P<0$, then the Volterra system (47) is dissipative with a global attractor $\mathcal{A}_{0}=\left\{\left(x_{j}(t)\right)=\mathbf{q}\right\} \subset C_{\gamma}^{+}$corresponding to the unique solution $\mathbf{q}$ of (53).

This proposition also holds when the interaction matrix $A$ is dissipative and the perturbation matrix $P<0$ is diagonal. In this case, the perturbation matriz $P D<0$ is also diagonal for any choice of gauge and we can choose the transformation such that $A D \leq 0$.

5.2. The case of a singular diagonal perturbation matrix. We now consider the more interesting case where the perturbation matrix $P$ is singular. We will only consider the case where $P$ is diagonal for simplicity although the analysis is easily extended to some other cases.

For completion we first consider the case $P=0$, for which the equations for $x_{j}$ decouple from the rest of the system and we obtain

$$
\begin{gathered}
\dot{x}_{j}=\varepsilon_{j} x_{j}+\sum_{k=1}^{m} a_{j k} x_{j} x_{k}, \\
\dot{y}_{j}=x_{j}-y_{j},
\end{gathered}
$$

for $j=1, \ldots, m$. In this case, (61) is just the Lotka-Volterra system (51), and $(62)$ can be integrated to obtain the solutions $y_{j}(t)$ as functions of $x_{j}(t)$.

For completeness we recall here the results of $[\mathbf{3}]$ for the LotkaVolterra system. Using (53), equations (61) become

$$
\dot{x}_{j}=x_{j} \sum_{k=1}^{m} a_{j k}\left(x_{k}-q_{k}\right), \quad j=1, \ldots, m .
$$

Then, on the set $\dot{V}=0$, from (58) and with the choice of interaction matrix given by the previous lemma, we obtain

$$
a_{j j}\left(x_{j}-q_{j}\right)=0, \quad j=1, \ldots, m .
$$

The diagonal entries of the interaction matrix $A \leq 0$ satisfy $a_{j j} \leq 0$. If we have $a_{j j}<0$ for all $j=1, \ldots, m$, from (59) and (64), it follows that

$$
x_{j}=y_{j}=q_{j}, \quad j=1, \ldots, m .
$$


Moreover, $A$ is nonsingular. In fact, if $A$ is a singular matrix there is a nonzero vector $\left(w_{1}, \ldots, w_{m}\right)$ such that $\sum_{k=1}^{m} a_{j k} w_{k}=0$ for $j=1, \ldots, m$. Then, the previous Proposition 3 implies that $a_{j j} w_{j}=0$ for all $j=$ $1, \ldots, m$ from which it follows that $a_{j j}=0$ for some $j$, a contradiction. Again we conclude that the solution $\mathbf{q}$ of (53) is unique. Therefore, also in this case if $\mathbf{q} \in \mathbb{R}_{+}^{m}$ system (50) is dissipative and possesses a global attractor which is a singleton, $\mathcal{A}=\{(\mathbf{q}, \mathbf{q})\}$.

If, on the other hand, we have $a_{j j}=0$ for some $j$, we need to use the original equation (50) to obtain more information regarding the dynamics on the set where $\dot{V}=0$. In this case, (59) still holds and we also have $x_{j}=q_{j}$ for some values of $j$. We split the variables $x_{j}$ into two groups that, upon reordering, correspond to $j \in\{1, \ldots, n\}$ and $j \in\{n+1, \ldots, m\}$ with $1 \leq n<m$. In the second group we include all the variables for which we have $x_{j}=q_{j}$ (either because $a_{j j}<0$ or due to a reduced graph argument involving the interaction matrix, see $[\mathbf{3}]$ for details). In the first group we include all the other variables $x_{j}$. Then, the solutions of (50) on the set $\dot{V}=0$ satisfy new equations of the form

$$
\dot{x}_{j}=x_{j} \sum_{k=1}^{n} \tilde{a}_{j k}\left(x_{k}-q_{k}\right), \quad j=1, \ldots, n
$$

and

$$
x_{j}=q_{j}, \quad j=n+1, \ldots, m,
$$

where the reduced interaction matrix $\tilde{A}=\left(\tilde{a}_{j k}\right)$ satisfies

$$
\tilde{a}_{j j}=0, \quad j=1, \ldots, n,
$$

and it is negative semidefinite, $\tilde{A} \leq 0$. These two conditions together imply that $\tilde{A}$ is skew-symmetric and the reduced interaction matrix is conservative. It follows from [3] that the reduced system (66) has a Hamiltonian formulation. This is contained in the next result that essentially corresponds to [3, Theorem 4.5].

Theorem 5. Consider the system of Lotka-Volterra equations (61) coupled with the linear equations (62) and assume that: (i) the LotkaVolterra system (61) has a singular point $\mathbf{q} \in \mathbb{R}_{+}^{m}$; and, (ii) the interaction matrix $A$ is stably dissipative. Then the dynamics of this system on the set $\dot{V}=0$ can be described by a Lotka-Volterra system (66) of dimension $n \leq m$ together with (67), and $y_{j}(t)=\int_{-\infty}^{0} e^{\theta} x_{j}(t+\theta) d \theta$ for $j=1, \ldots, m$. Moreover, the dynamics of (66) is Hamiltonian. 
The observation on $y_{j}$ arises from the integration of (62), for which the general solution has the form

$$
y_{j}(t)=C e^{-t}+\int_{-\infty}^{0} e^{\theta} x_{j}(t+\theta) d \theta .
$$

Moreover, this solution satisfies condition (49) of Theorem 1 if and only if $C=0$. In this case, if $x_{j}$ is a bounded solution then $y_{j}$ is also bounded. We remark that $x_{j}=q_{j}$ then implies $y_{j}=q_{j}$, therefore we have

$$
y_{j}=q_{j}, \quad j=n+1, \ldots, m,
$$

and if $x_{j}$ is periodic, then $y_{j}$ is also periodic but, in general, is out of phase.

We now consider the crucial case where $p_{j j}=0$ for $j=1, \ldots, l$ with $1 \leq l<m$, and $p_{j j}<0$ for $j=l+1, \ldots, m$. In this case, (58) and (50) imply that on the set where $\dot{V}=0$ we have

$$
y_{j}=x_{j}=q_{j}, \quad j=l+1, \ldots, m .
$$

Using (53), the equations (50) then become

$$
\left\{\begin{array}{l}
\dot{x}_{j}=x_{j} \sum_{k=1}^{l} a_{j k}\left(x_{k}-q_{k}\right), \quad j=1, \ldots, l, \\
\dot{y}_{j}=x_{j}-y_{j},
\end{array}\right.
$$

together with (71). Again the equations for $x_{j}, j=1, \ldots, l$, are decoupled and correspond to a Lotka-Volterra system. In fact, these equations can be written in the form

$$
\dot{x}_{j}=x_{j} \tilde{\varepsilon}_{j}+\sum_{k=1}^{l} a_{j k} x_{j} x_{k}, \quad j=1, \ldots, l,
$$

with

$$
\tilde{\varepsilon}_{j}=\varepsilon_{j}+\sum_{k=l+1}^{m} a_{j k} q_{k}, \quad j=1, \ldots, l .
$$

Moreover, from (53) and this last equation we obtain

$$
\tilde{\varepsilon}_{j}+\sum_{k=1}^{l} a_{j k} q_{k}=0, \quad j=1, \ldots, l,
$$

which ensures that $\tilde{\mathbf{q}}=\left(q_{1}, \ldots, q_{l}\right)$ is an equilibrium of $(73)$. 
If the reduced matrix is stably dissipative, with the proper choice of interaction matrix given by Lemma 4.2 in [3] (see also Lemma 3 in [14]), we obtain

$$
a_{j j}\left(x_{j}-q_{j}\right)=0, \quad j=1, \ldots, l .
$$

Therefore, by the previous argument, (73) can lead to a reduced system with a Hamiltonian structure.

However, (71) also implies the following equations

$$
\sum_{k=1}^{l} a_{j k}\left(x_{k}-q_{k}\right)=0, \quad j=l+1, \ldots, m,
$$

which, then constitute constraints that must be satisfied by the solutions of (48) on the set $\dot{V}=0$. Solving these equations, we determine $r$ variables $x_{j}$ in terms of the remaining $s=l-r$ variables, where $r$ denotes the rank of the reduced matrix $R=\left(a_{j k}\right)_{l+1 \leq j \leq m, 1 \leq k \leq l}$. With an eventual reordering of the variables we can assume that the first $s$ variables are free, and write

$$
x_{j}=q_{j}+\sum_{k=1}^{s} \beta_{j k}\left(x_{k}-q_{k}\right), \quad j=s+1, \ldots, l,
$$

where the coefficients $\left(\beta_{j k}\right)_{s+1 \leq j \leq l, 1 \leq k \leq s}$ depend on the entries of the matrix $R$. Then, we can write the first $s$ equations of (73) again in the form

$$
\dot{x}_{j}=x_{j} \varepsilon_{j}^{*}+\sum_{k=1}^{s} a_{j k}^{*} x_{j} x_{k}, \quad j=1, \ldots, s,
$$

with

$$
\varepsilon_{j}^{*}=\tilde{\varepsilon}_{j}+\sum_{k=s+1}^{l} a_{j k}\left(q_{k}-\sum_{i=1}^{s} \beta_{k i} q_{i}\right), \quad j=1, \ldots, s,
$$

and

$$
a_{j k}^{*}=a_{j k}+\sum_{i=s+1}^{l} a_{j i} \beta_{i k}, \quad j=1, \ldots, s, \quad k=1, \ldots, s .
$$

The remaining equations $(73)$, for $j=s+1, \ldots, l$, can also be written in the form (79) with $\varepsilon_{j}^{*}$ in the form (80) and $a_{j k}^{*}$ in the form (81). However, in general the compatibility with (78) imposes further restrictions that need to be satisfied by the solutions on the set $\dot{V}=0$. Therefore, we may obtain $x_{j}=q_{j}$ also for some $j=s, s-1, \ldots$, in which case we 
repeat the previous procedure. By iteration one eventually ends up with a reduced Lotka-Volterra system

$$
\dot{x}_{j}=x_{j} \varepsilon_{j}^{*}+\sum_{k=1}^{s_{0}} a_{j k}^{*} x_{j} x_{k}, \quad j=1, \ldots, s_{0},
$$

coupled with equations

$$
x_{j}=q_{j}+\sum_{k=1}^{s_{0}} \tilde{\beta}_{j k}\left(x_{k}-q_{k}\right), \quad j=s_{0}+1, \ldots, l,
$$

for the variables $x_{j}, j=1, \ldots, l$, together with the equations

$$
\dot{y}_{j}=x_{j}-y_{j}, \quad j=1, \ldots, l,
$$

for the variables $y_{j}, j=1, \ldots, l$, and

$$
x_{j}=y_{j}=q_{j}, \quad j=l+1, \ldots, m,
$$

for all the remaining variables. Summarizing these results we have the following

Theorem 6. Consider the Volterra system (47) and assume that:

(i) there exists an equilibrium solution with $\mathbf{q} \in \mathbb{R}_{+}^{m}$ given by (53); and

(ii) the perturbation matrix $P$ is diagonal and singular, with

$$
p_{j j}=0, j=1, \ldots, l, \quad p_{j j}<0, \quad j=l+1, \ldots, m .
$$

Then the dynamics of (47) can be described by a Lotka-Volterra system (82) of dimension $s_{0} \leq l$ together with equations (83).

Moreover, $y_{j}(t)=\int_{-\infty}^{0} e^{\theta} x_{j}(t+\theta) d \theta$ for $j=1, \ldots, m$.

Then, the results of [3] apply again to the Lotka-Volterra system (82), eventually further reducing the dimension $s_{0}$, and we obtain the following

Corollary 7. If in addition to the hypothesis (i) and (ii) of Theorem 6, we assume that the reduced interaction matrix $A^{*}=\left(a_{j k}^{*}\right)_{1 \leq j \leq s_{0}, 1 \leq k \leq s_{0}}$ is stably dissipative, then the dynamics of (47) can be described by a reduced Lotka-Volterra system (82) with dimension $s_{0} \leq l$ together with equations (83), and the dynamics of (82) is Hamiltonian.

The Hamiltonian formulation of (66) or (82) leads to a very rich and complex behavior for the solutions of (47). To support this observation we mention again that $[\mathbf{3}]$ presents an example for which the Hamiltonian system for (66) is non-integrable and the flow contains families of strongly hyperbolic periodic orbits. 


\section{Recent results.}

An interesting monograph is the Ph.D. Thesis, in Portuguese, of Telmo Peixe [18] that gives emphasis to the dissipative Lotka-Volterra systems.

Another paper by Duarte and Peixe deals with a new concept: the rank of stably dissipative graphs, see [4]; they show that the rank of the defining matrix, which is the dimension of an associated foliation, is completely determined by the system's graph.

The paper [31] by Zhao and Luo proves necessary and sufficient conditions for a matrix to be stably dissipative. Then, a graph-theoretic classification method for stably dissipative matrices is proposed, based on which, for all 5-order stably dissipative matrices, the associated graphs are completely classified as 27 topologically different graphs and for each graph the dynamics of the corresponding Lotka-Volterra systems are discussed.

\section{Acknowledgements}

We never forget that J. Hale called our attention for the works of Redheffer et al. during the Equadiff 95 conference held in Lisbon.

\section{References}

[1] V. I. ARNOL'D, "Mathematical methods of classical mechanics", Translated from the Russian by K. Vogtmann and A. Weinstein, Second edition, Graduate Texts in Mathematics 60, Springer-Verlag, New York, 1989.

[2] J. Coste, J. Peyraud, and P. Coullet, Asymptotic behaviors in the dynamics of competing species, SIAM J. Appl. Math. 36(3) (1979), 516-543. DOI: 10.1137/0136039.

[3] P. Duarte, R. L. Fernandes, and W. M. Oliva, Dynamics of the attractor in the Lotka-Volterra equations, J. Differential Equations 149(1) (1998), 143-189. DOI: 10.1006/jdeq.1998.3443.

[4] P. Duarte And T. Peixe, Rank of stably dissipative graphs, Linear Algebra Appl. 437(10) (2012), 2573-2586. DOI: 10.1016/j.laa. 2012.06 .015 .

[5] J. HALE, "Theory of functional differential equations", Second edition, Applied Mathematical Sciences 3, Springer-Verlag, New YorkHeidelberg, 1977.

[6] Y. Hino, S. Murakami, and T. Naito, "Functional-differential equations with infinite delay", Lecture Notes in Mathematics 1473, Springer-Verlag, Berlin, 1991. 
[7] M. W. Hirsch, Systems of differential equations which are competitive or cooperative: III. Competing species, Nonlinearity 1(1) (1988), 51-71. DOI : 10.1088/0951-7715/1/1/003.

[8] M. W. Hirsch, Systems of differential equations which are competitive or cooperative: I. Limit sets, SIAM J. Math. Anal. 13(2) (1982), 167-179. DOI : 10.1137/0513013.

[9] J. Hofbauer And K. Sigmund, "The theory of evolution and dynamical systems", Mathematical aspects of selection, Translated from the German, London Mathematical Society Student Texts 7, Cambridge University Press, Cambridge, 1988.

[10] G. IsRAEL, Volterra's "analytical mechanics" of biological associations. I, Arch. Internat. Hist. Sci. 41(126) (1991), 57-104.

[11] J. P. LASAlLE, An invariance principle in the theory of stability, in: "Differential Equations and Dynamical Systems" (Proc. Internat. Sympos., Mayaguez, P.R., 1965), Academic Press, New York, 1967, pp. 277-286.

[12] A. J. LotKA, "Elements of Physical Biology", Baltimore, Williams \& Wilkins Co., 1925.

[13] J. Milnor, "Morse theory", Based on lecture notes by M. Spivak and R. Wells, Annals of Mathematics Studies 51, Princeton University Press, Princeton, N.J. 1963.

[14] W. M. Oliva And C. Rocha, Reducible Volterra and Levin-Nohel retarded equations with infinite delay, J. Dynam. Differential Equations 22(3) (2010), 509-532. DOI: 10.1007/s10884-010-9177-y.

[15] W. M. Oliva, J. S. Dos Santos, and P. Z. Táboas, A set of global bounded solutions for a Volterra system of retarded equations on $\mathbb{R}_{+}^{3}$, NoDEA Nonlinear Differential Equations Appl. 7(3) (2000), 259-283. DOI: 10.1007/PL00001425.

[16] W. M. Oliva And P. TÁBoAs, Existence of periodic orbits, set of global solutions and behavior near equilibrium for Volterra equations of retarded type, Portugal. Math. 54(2) (1997), 165-186.

[17] P. J. OLver, "Applications of Lie groups to differential equations", Graduate Texts in Mathematics 107, Springer-Verlag, New York, 1986. DOI: $10.1007 / 978-1-4684-0274-2$.

[18] T. Peixe, Sistemas Lotka-Volterra Dissipativos, Dissertation, UL., Fac. de Ciências, D. Matemática (2010).

[19] M. Plank, Hamiltonian structures for the $n$-dimensional LotkaVolterra equations, J. Math. Phys. 36(7) (1995), 3520-3534. DOI: $10.1063 / 1.530978$. 
[20] H. Poincaré, "Les méthodes nouvelles de la mécanique céleste". Tome I. Solutions périodiques. Non-existence des intégrales uniformes. Solutions asymptotiques, Paris, Gauthier-Villars et Fils, 1892.

[21] R. ReDhefFer, A new class of Volterra differential equations for which the solutions are globally asymptotically stable, J. Differential Equations 82(2) (1989), 251-268. DOI: 10.1016/0022-0396(89) 90133-2.

[22] R. Redheffer, Volterra multipliers. I, II, SIAM J. Algebraic Discrete Methods 6(4) (1985), 592-611, 612-623. DOI: 10.1137/ 0606059. DOI: 10.1137/0606060.

[23] R. Redheffer And W. Walter, Solution of the stability problem for a class of generalized Volterra prey-predator systems, J. Differential Equations 52(2) (1984), 245-263. DOI: 10.1016/00220396 (84) 90179-7.

[24] R. RedhefFer And Z. M. Zhou, A class of matrices connected with Volterra prey-predator equations, SIAM J. Algebraic Discrete Methods 3(1) (1982), 122-134. DOI: 10.1137/0603012.

[25] R. Redheffer and Z. M. Zhou, Global asymptotic stability for a class of many-variable Volterra prey-predator systems, Nonlinear Anal. 5(12) (1981), 1309-1329. DOI : 10.1016/0362-546X (81) 90108-5.

[26] S. Smale, On the differential equations of species in competition, J. Math. Biol. 3(1) (1976), 5-7.

[27] V. Volterra, Variazione e fluttuazioni del numero d'individui in specie animali conviventi, Mem. Accad. Lincei 2 (1926), 31-113.

[28] V. Volterra, Sur la théorie mathématique des phénomènes héréditaires, Journ. de Math. (9) 7 (1928), 249-298.

[29] V. VolterRA, "Lecons sur la théorie mathématique de la lutte pour la vie", Paris, Gauthier-Villars, 1931.

[30] M. L. ZEEMAN, Extinction in competitive Lotka-Volterra systems, Proc. Amer. Math. Soc. 123(1) (1995), 87-96. DOI: 10.2307/ 2160613.

[31] X. ZhaO AND J. Luo, Classification and dynamics of stably dissipative Lotka-Volterra systems, International Journal of Non-Linear Mechanics 45(6),(2010), 603-607. DOI: 10.1016/j.ijnonlinmec. 2009.07 .006 .

Center for Mathematical Analysis, Geometry and Dynamical Systems - CAMGSD

Av. Rovisco Pais

1049-001 Lisboa

Portugal

E-mail address: wamoliva@math.ist.utl.pt 